\section{Biodiversity treaty misguided}

SIR - The 1992 Convention on Biological Diversity addresses a broad spectrum of issues related to the protection of biological diversity. These include the conservation of habitats in developing nations and the availability of resources to make this possible (see D. Putterman Nature 371, 553; 1994). This 'Biodiversity Treaty' was conceived as an unprecedented opportunity for industrialized and developing countries to reconcile issues of conservation and access to biological resources. But it is burdened with the problematic question of an international biosafety protocol (regulations) for biotechnology, which in any case has little to do with biodiversity. The kind of biosafety regulation being contemplated - for example, for field trials of improved varieties of potatoes, maize, rice or cassava - would discourage innovative research and development, commit the countries concerned to a poorly conceived highest-common-denominator level of regulation, and deprive domestic regulatory agencies of discretion in setting regulatory policy.

The international bureaucrats discussing the form and implementation of a biosafety protocol reveal their misguided intentions clearly in an official description of the April 1993 proceedings of an expert panel established to implement these aspects of the treaty (Expert Panels Established to Follow-Up on the Convention on Biological Diversity, Report of Panel IV, UNEP/Bio.Div/Panels/Inf. 1, 28 April 1993). According to paragraphs 57 and 58 "a majority of the Panel members believed the organisms covered by a possible protocol should be restricted to genetically modified organisms", along the lines of the European Union's approach, which defines what is regulated not by riskrelated criteria, but according to whether molecular techniques of genetic manipulation have been used.

The panel notes that the scope of regulation "does not include organisms modified by traditional breeding methods", regardless of pathogenicity, likelihood of constituting an environmental nuisance, or other potential risks. This approach has been widely discredited, the scientific consensus holding that there is no conceptual difference between organisms crafted with older techniques (such as mutagenesis or hybridization) and the newer molecular techniques (such as genesplicing), and that regulation should be risk-based. The panel took little notice of a minority viewpoint cited in the report that the scope of regulation proposed was unscientific and "would ignore organisms actually known to present a threat to biodiversity, while focusing on others for which only hypothetical analyses can be offered". (In support of the minority view, consider that the bacteria that cause anthrax or bubonic plague could be tested - for example, as a pesticide - without regulation, while a field trial of a genespliced extended shelf-life tomato would require a government review.)

The wrong-headed regulation in the making would hurt research and development in ways not limited to the developing world, or even to international transfers and transactions. Paragraphs 74 and 75 of the panel report observe ominously that "a majority of the Panel members interpreted the [treaty] language . . . as if both international transfer of organisms and domestic handling and use of organisms were covered. The majority of the Panel members thought that domestic regulation should be covered by a possible protocol."

The primary goals of the treaty are laudable but the mechanisms provided to accomplish them are vague or impotent, while the biosafety protocol is an imminent hazard to the diffusion of biotechnology through the developing world. Its ability to enhance safety in the developing world is extremely doubtful; and the likelihood of its being cost-effective is nil. Moreover, the protocol's possible jurisdiction over strictly domestic activities could effectively take away individual nations' ability to adopt rational policies, thus discouraging innovation. Ironically, the protocol would stifle development of environmentally friendly biotech innovations that can help clean up toxic wastes, purify water and displace agricultural chemicals - products especially needed in the developing world. The treaty is far more likely to cause harm than to make significant inroads towards its goals. It should be abandoned and a new, sound one renegotiated.

Henry I. Miller

Hoover Institution and

Institute for International Studies,

Stanford University,

Stanford, California 94305-6010, USA

\section{Athletic nonsense}

SIR - This has been a remarkable season for athletic track events. Already the 100 -metre world record has been broken by $0.01 \mathrm{~s}$ while the 10,000 -metre world record is now $26 \mathrm{~min} 52.23 \mathrm{~s}$. Presumably a runner who completes the 10,000 metres in $26 \mathrm{~min} 52.22 \mathrm{~s}$ will be judged to be the new world record holder. I suggest that the measurement of track events to 0.01 seconds is a nonsense and that there is a need for the time measurement to be commensurate with the errors associated with the several variables and assumptions involved in such measurements which should be familiar to any scientist.

A 10 -second 100 -metres runner covers $10 \mathrm{~cm}$ in 0.01 seconds while a 10,000 metres runner covers just over $6 \mathrm{~cm}$ in the same time. The winner is determined by detection of the intersection of a part the body from the torso to neck with the finish line. A wind assistance of less than 2 metres per second is permitted. It is highly unlikely that the length of the 10,000 metre track can be measured with an accuracy to anything like $6 \mathrm{~cm}$. Even thermal expansion of the track will produce comparable errors. For a 25-lap 10,000 -metre race, an error of $6 \mathrm{~cm}$ corresponds to a systematic error of $2.4 \mathrm{~mm}$ in the distance for one lap.

It is assumed that the athletes respond instantaneously to the starter's signal. But within the margin of 0.01 seconds some of the athletes may have anticipated the starter's signal and already covered several centimetres. It is unrealistic to attach any significance to differences in timings of 0.01 seconds. And it is probably unrealistic to quote 100 -metre results to better than $0.1 \mathrm{~s}$ and for the 10,000 -metre race to better than $1 \mathrm{~s}$. In the latter case the uncertainty may even reach a few seconds.

I do not believe that any scientific significance can be attached to the claim that the new 100-metre world record holder ran faster than his predecessor, indeed he may even have been slower.

\section{B. W. Wybourne}

Instytut Fizyki, Uniwersytet Mikolaja

Kopernika,

ul. Grudziadzka 5/7, 87-100 Toruń, Poland

\section{Counting time}

SIR - Kukla's communication (Nature 372, 124; 1994) is a classic example of the confusion that the $\mathrm{BC} / \mathrm{AD}$ system of reckoning time generates, even among university professors expert in chronology. The fact is that the time interval between 1.5 years $\mathrm{BC}$ and 1.5 years $\mathrm{AD}($ sic $)$ is not commensurate with that between the dates 1.5 BC and AD 1.5. Because the years run backward in the $\mathrm{BC}$ numeration while time runs forward, and because there is no year zero, there are six months between $00.00 \mathrm{~h} 1 \mathrm{July}, 1 \mathrm{BC}(-1.5 \mathrm{BC})$ and $00.00 \mathrm{~h} 1$ January, $A D$ 1; and six more months between $00.00 \mathrm{~h} 1 \mathrm{January}$ and $00.00 \mathrm{~h} 1$ July, $\mathrm{AD} 1$ (- $\mathrm{AD} 1.5)$. Therefore, there is only one year between $1.5 \mathrm{BC}$ and $\mathrm{AD} 1.5$, as I said in my original communication. Notice also that the limit of 1.999 . . . BC is $\mathrm{AD} 1$, not $2 \mathrm{BC}$. The calendar reform I have proposed (Nature 366, 716; 1993 and EOS $75,219 ; 1994)$ will clear the confusion. Cesare Emilian! Department of Geological Sciences, University of Miami,

Coral Gables, Florida 33124, USA 